\title{
Simple New Test for Rapid Differentiation of Prototheca wickerhamii from Prototheca zopfii
}

\author{
MANUEL J. CASAL* AND JUAN GUTIERREZ \\ Department of Microbiology, School of Medicine, Cordoba University, Cordoba, Spain
}

Received 9 February 1983/Accepted 1 July 1983

\begin{abstract}
A simple new test to differentiate Prototheca wickerhamii from Prototheca zopfii by determining susceptibility to clotrimazole is described. A $50-\mu \mathrm{g}$ clotrimazole disk provides a rapid and reliable means of distinguishing $P$. wickerhamii from $P$. zopfii.
\end{abstract}

Prototheca wickerhamii and Prototheca zopfii are two species of achlorophyllous algae that are known to be opportunistic pathogens of humans $(3,4,5)$. Separation of the two species is based on macroscopic and microscopic examination and sugar and alcohol assimilation patterns. Immunofluorescence may be used for the confirmation of these two species (6).

In this communication, we describe a possible new differentiating test based on the susceptibility of each species to clotrimazole.

Forty-four strains of $P$. wickerhamii, 10 strains of Prototheca stagnora and 21 strains of $P$. zopfii were included in the study. The cultures were received from the collections of $L$. Ajello, Centers for Disease Control, Atlanta, Ga., E. H. Ball, University of Glasgow, Scotland; M. Feo, Instituto de Medicina Tropical, Universidad Central de Venezuela, Caracas, Venezuela; P. Hocquet, Service des Maladies Parasitaires et Exotiques, Centre Hospitalier Regional, France; H. Koenig, Institut de Parasitologie et Pathologie Tropical, Université L. Pasteur, Strasbourg, France; C. P. Kurtzman, Agricultural Research Service Culture Collection, Peoria, Ill.; R. S. Pore, Department of Microbiology, West Virginia University, Morgantown, West Virginia; and from our own laboratory (2). The $P$. stagnora strains originated from sewage water, the collections of $P$. zopfii from sewage water, feces, and finger nails, and the $P$. wickerhamii strains from sewage water, skin, and human blood.

Identification of $P$. wickerhamii and $P$. zopfii isolates was confirmed by macroscopic and microscopic examination, and by determining their abilities to use sucrose, trehalose, lactose, inositol, $n$-propanol, and xylose as carbon sources for growth (1). Confirmation of the species identification was made by W. Kaplan, Division of Mycotic Diseases, Centers for Disease Control, by immunofluorescence (6).
The disks used in this study contained $50 \mu \mathrm{g}$ of clotrimazole. Disk diffusion tests were made on 20-ml of Sabouraud glucose agar (Difco Laboratories) in petri $(90 \mathrm{~mm})$ dishes inoculated with an even suspension of algal cells. The Prototheca inoculum was prepared in distilled water with organisms that were incubated for 7 days at $37^{\circ} \mathrm{C}$ on Sabouraud glucose agar. The suspensions were adjusted to a no. 1 MacFarland standard before inoculating the plates. Plates were examined on days 2 and 5 after inoculation. All tests were repeated at least twice to confirm reproducibility. Strains were considered resistant if growth was not inhibited by clotrimazole. Inhibition was defined as a zone of inhibition of $\mathbf{1 0}$ $\mathrm{mm}$ or more.

All strains of $P$. zopfii were resistant to clotrimazole (Table 1). In contrast, all strains of $P$. wickerhamii were susceptible, with an average inhibitory zone diameter of $23 \mathrm{~mm}$.

The results of this study show that clotrimazole can be used for aiding in the separation of $P$. wickerhamii from $P$. zopfii. Susceptibility to this agent is markedly different between these two species. With a concentration of $50 \mu \mathrm{g}$ of clotrimazole per disk, Prototheca spp. inhibited at $37^{\circ} \mathrm{C}$ can be presumed to be $P$. wickerhamii, whereas resistant strains are likely to be $P$. zopfii. All strains of $\boldsymbol{P}$. stagnora tested were susceptible to clotrimazole, but $P$. stagnora does not grow at $37^{\circ} \mathrm{C}$.

TABLE 1. Activity of clotrimazole ( $50 \mu \mathrm{g}$ per disk) against $\boldsymbol{P}$. wickerhamii, $\boldsymbol{P}$. zopfii, and $\boldsymbol{P}$. stagnora

\begin{tabular}{lcccc}
\hline \multirow{2}{*}{ Species } & $\begin{array}{c}\text { No. of } \\
\text { strains } \\
\text { tested }\end{array}$ & $\begin{array}{c}\text { No. (\%) } \\
\text { of strains } \\
\text { inhibited }\end{array}$ & \multicolumn{2}{c}{$\begin{array}{c}\text { Avg diam (mm) } \\
\text { of inhibitory }\end{array}$} \\
\cline { 3 - 5 } & & & Range & Mean \\
\hline$P$. wickerhamii & 44 & $44(100)$ & $10-36$ & 23 \\
$P$. zopfi & 21 & $0(0)$ & 0 & 0 \\
$P$. stagnora & 10 & $10(100)$ & $10-30$ & 20 \\
\hline
\end{tabular}


We thank L. Ajello, E. H. Ball, M. Feo, P. Hocquet, H. Koenig, C. P. Kurtzman, and R. S. Pore for providing some of the cultures used in this study, W. Kaplan for the confirmation of identity by immunofluorescence of our isolates, and $\mathrm{L}$. Ajello for his helpful advice and continued collaboration with us in the Prototheca studies.

\section{LITERATURE CITED}

1. Arnold, P., and D. G. Ahearn. 1972. The systematics of the genus Prototheca with a description of a new species $P$. filamenta. Mycologia 64:265-275.
2. Casal, M., and F. Solis. 1981. First isolation of Prototheca species in Spain. Mycopathology 74:55-56.

3. Davies, R. R., H. Spencer, and P. D. Wakelin. 1964. A case of human protothecosis. Trans. Roy. Soc. Trop. Med. Hyg. 58:448-453.

4. Kaplan, W. 1977. Protothecosis and infections caused by morphologically similar green algae, p. 218-232. In Proceedings of the Fourth International Conference on the Mycoses. PAHO. Sci. Publ. no. 356

5. Sudman, M. S. 1974. Protothecosis: a critical Review. Am. J. Clin. Pathol. 61:10-19.

6. Sudman, M. S. 1973. Identification of the Prototheca species by immunofluorescence. Appl. Microbiol. 25:981-990. 